Oper Orthop Traumatol $2011 \cdot 23: 253-253$

DOI 10.1007/s00064-011-0152-5

Online publiziert: 18. September 2011

(c) Springer-Verlag 2011

A. Eckardt

Hirslanden-Klinik Birshof, Münchenstein

\title{
Amputationen an der unteren Extremität
}

Wir hoffen, mit den Beiträgen in dieser Ausgabe eine Übersicht über die Techniken der häufigsten Amputationen und der jeweiligen Nachbehandlung geben zu können. Die wegweisenden Publikationen und die weitere unermüdliche Länder- und Kontinente übergreifende Edukation zum Thema Amputationen und Prothesenversorgung erfolgen seit Jahrzehnten richtungweisend durch René Baumgartner. Wir sind dankbar, dass er die Aufgabe übernahm, eine aktuelle Zusammenstellung der Amputationstechniken am Vor- und Mittelfuß, Unterund Oberschenkel sowie der Knieexartikulation zu geben. Das schwierige Kapitel der Rückfußamputationen beleuchtet S. Rammelt und C. Jacobs reflektiert die Notfallamputationen nach schwerem Trauma.

Die Indikationsstellung allerdings, wann und in welcher Höhe bei z. B. einem Patienten mit diabetischem Fußsyndrom oder pAVK eine Amputation durchgeführt werden muss, bleibt weiterhin Ergebnis des intensiven Austauschs von Internisten, Angiologen, Radiologen, Gefäßchirurgen und dem operierenden Orthopäden bzw. Unfallchirurgen. Im Einzelfall mag die Einschätzung „life before limb“ noch zutreffen, moderne interdisziplinäre Konzepte und die Kenntnis schonender, wohlgeplanter Operationstechniken ermöglichen es jedoch häufig, die Extremität zu erhalten und somit einen erheblichen Gewinn an Mobilität,
Lebensqualität und -erwartung für den Patienten zu gewährleisten.

\section{จ Moderne interdisziplinäre Konzepte ermöglichen häufig den Erhalt einer Extremität}

Dies ist nur in einem ausgewogenen $\mathrm{Zu}$ sammenspiel von nicht operativ und operativ tätigen Kollegen und unter entsprechender Mitarbeit der medizinischen Assistenzberufe in aufeinander abgestimmten Strukturen möglich. Vielleicht kann dieser Themenschwerpunkt auch einen Beitrag dazu leisten, die hierfür notwendigen administrativen und organisatorischen Strukturen für ein kollegiales interdisziplinäres Vorgehen erfolgreicher zu gestalten.

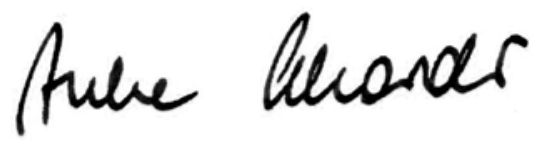

Anke Eckardt

Korrespondenzadresse

Prof. Dr. A. Eckardt

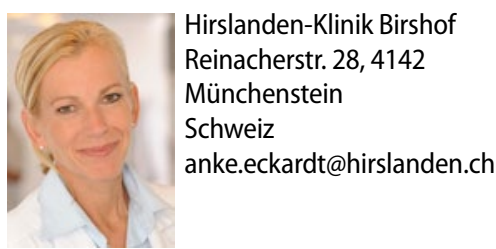

des Operateurs und des interdisziplinären Behandlungsteams ab. 